\title{
Potencial de Uso de um Tanque Solar de Gradiente Salino para o Armazenamento de Energia Térmica
}

\section{Potential Use of a Salt Gradient Solar Pond to Store Thermal Energy}

\author{
Daniel Vinícius S. Veras ${ }^{1}$; Rafael da C. I. Fontan²; Lizzy Ayra A. Veríssimo³; Ana \\ Paula F. Machado ${ }^{4}$; Cecília A. Mourão ${ }^{5}$, Renata Cristina F. Bonomo ${ }^{6}$
}

\section{Resumo}

A demanda por fontes energéticas que permitam um desenvolvimento sustentável tem impulsionado estudos sobre a obtenção de energias alternativas, renováveis e não poluentes como a energia hídrica, eólica, biomassa e solar. Sendo assim, o objetivo neste trabalho foi avaliar o potencial de um tanque solar de gradiente salino (TSGS) para o armazenamento de energia térmica obtida a partir da energia solar. Um TSGS piloto com área superficial de $1,76 \mathrm{~m}^{2}$ e volume de $1 \mathrm{~m}^{3}$ foi montado no município de Itapetinga, BA e a temperatura nas camadas convectivas superior (CCS) e inferior (CCI) foi monitorada entre 20 de agosto e 03 de novembro de 2008. Verificou-se que a diferença de temperatura entre as camadas aumentou de $2^{\circ} \mathrm{C}$ para $13,2^{\circ} \mathrm{C}$, com um valor médio de $5,7^{\circ} \mathrm{C}$ para o período. Para uma eficiência de $30 \%$ na utilização da energia acumulada, seria possível elevar em $10^{\circ} \mathrm{C}$ a temperatura de $50 \mathrm{~L}$ de leite resfriado em um laticínios. Em períodos mais quentes a diferença de temperatura poderia ser ainda maior. Os resultados obtidos neste trabalho demonstram o potencial de utilização em nosso país do TSGS para armazenar energia térmica a partir da energia solar, como alternativa renovável e não-poluente, para indústrias e propriedades rurais.

Palavras-chave: Fonte alternativa. Camadas convectivas. Energia renovável. Sal. Sustentabilidade.

\begin{abstract}
Demand for energy sources that allow a sustainable development has stimulated studies on the acquisition of alternative energy, renewable and clean such as hydro, wind, biomass and solar energies. Therefore, the objective in this work was to evaluate the potential of a Salt Gradient Solar Pond (SGSP) in order to obtain thermal energy from solar energy. A pilot solar pond with 1,76 $\mathrm{m}^{2}$ of surface area and $1 \mathrm{~m}^{3}$ of volume was set up in Itapetinga, Bahia, and temperatures in upper and lower convective layers were monitored between August 20 and November 3, 2008. It was found that the temperature difference between the layers increased from $2^{\circ} \mathrm{C}$ to $13.2^{\circ} \mathrm{C}$, with a medium value of $5.7^{\circ} \mathrm{C}$ for the period. Considering a $30 \%$ efficiency for utilization of stored energy, it would be possible increase by
\end{abstract}

1 Mestrando em Engenharia de Alimentos, Universidade de São Paulo; daniel.vsveras@gmail.com

2 Docente do Departamento de Tecnologia Rural e Animal da Universidade Estadual do Sudoeste da Bahia; rafaelfontan@yahoo. com.br

3 Mestre e Doutoranda em Ciência e Tecnologia de Alimentos, Universidade Federal de Viçosa; lizzy.alcantara@gmail.com

${ }^{4,5}$ Graduandas em Engenharia de Alimentos, Universidade Federal de Viçosa; anapfmachado@hotmail.com; cecilia_mourao@ yahoo.com.br

6 Pesquisadora nível 2 do CNPq e Docente do Departamento de Tecnologia Rural e Animal da Universidade Estadual do Sudoeste da Bahia; bonomorcf@pq.cnpq.br 
$10^{\circ} \mathrm{C}$ the temperature of $50 \mathrm{~L}$ of chilled milk in a dairy industry, thus demonstrating its potential. In the end of Spring or Summer the temperature difference would be superior. Results demonstrate the potential use of solar ponds by industries and farms in our country, as a renewable and non-polluting alternative, being able to store thermal energy from solar energy.

Key words: Alternative source. Convective layers. Renewable Energy. Salt. Sustainability.

\section{Introdução}

A necessidade de se obter fontes energéticas que permitam um desenvolvimento sustentável tem impulsionado estudos sobre a obtenção de energias alternativas, renováveis e não poluentes como a energia hídrica, eólica, biomassa e solar. Dentre as fontes de energia citadas, uma das mais abundantes é a energia solar, que ainda não é plenamente explorada (JANNUZZI, 2000). Assim, existe uma demanda pelo desenvolvimento de tecnologias de baixo custo para sistemas de aquecimento solar e que também possam ser aplicados em processos industriais (OUNI et al., 2003).

Um tanque solar é um corpo de água que armazena a energia térmica a partir da energia solar. A energia solar aquece um corpo de água, que está exposto ao sol, esta é dissipada na forma calor a menos que algum método seja utilizado para armazená-lo. A água aquecida pelo sol se expande e ascende à medida que se torna menos densa. Uma vez que esta alcança a superfície, perde a energia armazenada na forma de calor para o ar, por meio de convecção ou evaporação, dissipando energia térmica neste processo. A água mais fria, que é mais densa, move-se para baixo para ocupar o volume deslocado de água quente, criando uma circulação convectiva natural que mistura a água e dissipa energia na forma de calor. O design de tanques solares reduz tanto a convecção quanto a evaporação de maneira a armazenar a energia térmica (AGHA; ABUGHRES; RAMADAN, 2002), (OUNI et al., 2003), (GIESTAS et al., 2009).

Existem duas categorias principais de tanques solares: os tanques não convectivos, que reduzem a perda de energia térmica prevenindo a ocorrência de convecção dentro do tanque; e os tanques convectivos, que reduzem a perda de energia térmica evitando a evaporação com uma cobertura sobre a superfície do tanque (TABOR, 1981), (RIFFE; LEITE; BELO, 2007).

Dentre os tanques não convectivos, podemos citar o tanque solar de gradiente salino e o tanque de membranas (GIESTAS; JOYCE; PINA, 1997), (HULL, 1980). Um tanque solar de gradiente salino (TSGS) possui três camadas bem definidas com diferentes concentrações de sal, a saber, camada convectiva superior (CCS), camada não convectiva (CNC) e camada convectiva inferior (CCI) (OUNI et al., 2003), (AGHA; ABUGHRES; RAMADAN, 2002), (ARREBOLA et al., 2010). Os sais mais comumente usados são os cloretos de sódio e magnésio e nitrato de sódio (VELMURUGAN; SRITHAR, 2008). O aumento da temperatura é proveniente da radiação solar, transferido para as camadas inferiores na forma de energia térmica (ARREBOLA et al., 2010).

Tanques solares de gradiente salino são economicamente viáveis apenas onde há abundância de sal barato e de boa qualidade, grandes áreas de terra plana, fácil acesso à água e clima temperado ou tropical (ALAGAO, 1996). Fatores ambientais são igualmente importantes, como por exemplo, a necessidade de prevenção da contaminação do solo e águas adjacentes pela salmoura do tanque solar (KISHORE; KUMAR, 1996). Por estas razões o Brasil se constitui como um candidato promissor para o desenvolvimento desta tecnologia.

A utilização de tanques solares de gradiente salino vem sendo estudada em diversos países, como Israel, Índia, Estados Unidos, Austrália, Líbia, Tunísia, Turquia, Jordânia e Filipinas (DOR; EHRUC, 1987), (OUNI et al., 2003), (SHAH; SHORT; 
FYNN, 1981), (TABOR, 1981). A menção destes países nos remete ao primeiro fator decisivo para o desempenho do tanque solar de gradiente salino, o fator climático, uma vez que estes países possuem pelo menos uma região abundante em exposição solar por algum período do ano. No Brasil, apesar do potencial existente devido às condições climáticas favoráveis, estudos envolvendo TSGS's ainda são escassos e pontuais (LOBO; ARAÚJO Jr., 1984; DHERE et al., 2005; JORGE; FADIGAS, 2011;).

As aplicações do tanque solar incluem aquecimento comunitário, residencial e comercial, processos térmicos agroindustriais que envolvam baixas temperaturas, pré-aquecimento para aplicações industriais e geração de eletricidade (HASNAIN, 1998), (RABL, 1985), (LU et al., 2004). Dentre as principais vantagens de se utilizar energia solar a partir de um tanque solar de gradiente salino, destacam-se o armazenamento contínuo de calor, a grande área de coleta de calor a um baixo custo e a adequabilidade tanto para geração de energia térmica como elétrica (LU et al., 2004), (TABOR, 1981).

Sendo assim, o objetivo deste trabalho foi avaliar o potencial de armazenamento de energia térmica obtida a partir da energia solar em um protótipo de TSGS produzido com materiais de baixo custo, visando à futura aplicação em processos industriais.

\section{Material e métodos}

\section{Construção e localização do tanque solar de gradiente salino}

Para a construção do TSGS utilizou-se um tanque retangular de fibrocimento com capacidade volumétrica de $1000 \mathrm{~L}, 115 \mathrm{~kg}$ de cloreto de sódio comercial, um tubo de cloreto de polivinila (PVC) com $10 \mathrm{~mm}$ de diâmetro e $1 \mathrm{~m}$ de comprimento e adaptadores para mangueiras de borracha. A superfície externa e interna do tanque solar foi pintada com tinta acrílica fosca na cor preto.

O tanque solar foi posicionado em uma área de campo aberto, sem vegetação ou construções próximas que pudessem fazer sombra sobre ele, de forma que a sua própria sombra projetada dentro do tanque fosse a menor possível. Para isso sua maior dimensão foi alinhada no sentido lesteoeste e o tanque sofreu uma leve inclinação $\left(\sim 10^{\circ}\right)$ para posicionar seu fundo o mais perpendicular possível à trajetória descrita pelo sol. Alguns TSGS experimentais de pequeno porte localizados acima do nível do solo vêm sendo relatados por diversos autores (OULD DAH ET AL., 2010; TUNDEE et al., 2010; LEBLANC et al., 2011; MALIK et al., 2011).

O tanque esteve localizado no município de Itapetinga, Bahia, no campus da Universidade Estadual do Sudoeste da Bahia, exatamente na latitude $15^{\circ} 15^{\prime} 17,5^{\prime}$ ' S (sul), longitude $40^{\circ} 16^{\prime}$ $12,8^{\prime}$ W (oeste) e altitude de $279 \mathrm{~m}$ em relação ao nível do mar, no período de agosto a novembro de 2008, do meio do inverno ao meio de primavera. Itapetinga é um município com ventos de até $4 \mathrm{~m} / \mathrm{s}$ e incidência de radiação solar em torno de $2800 \mathrm{~kJ} / \mathrm{m}^{2}$ em horários de pico (INMET, 2012).

O TSGS foi preenchido com água até aproximadamente um terço de seu volume, sendo nela dissolvidos $70 \mathrm{~kg}$ de cloreto de sódio para a formação da camada conectiva inferior (CCI). A dissolução do sal foi realizada de forma empírica, até que se constatasse a presença de excesso de sal no fundo do tanque, evidenciando assim o ponto de saturação daágua para a temperatura de operação. Um tubo de PVC foi colocado na parte interna do tanque, próximo a uma das quinas do mesmo, de modo que uma das extremidades ficasse a $2 \mathrm{~cm}$ do fundo e a outra ficasse acima da linha d'água do tanque cheio. Por meio dele era possível se adicionar mais sal à CCI à medida que sua temperatura aumentasse, sem perturbar as demais camadas formadas.

Posteriormente, preencheu-se o restante do tanque solar com água a uma baixa vazão, aproximadamente $1,2 \times 10^{-5} \mathrm{~m}^{3} / \mathrm{s}$, até que a mesma começasse a escoar pela válvula de saída. Tal vazão de preenchimento 
deve ser reduzida para não provocar turbulências nas camadas de água formadas no tanque solar durante este processo, visto que é importante a existência das três camadas bem definidas. Nesta etapa do preenchimento, ocorre a formação da interface entre a CCI, onde se encontra a maior quantidade de sal, e a e a camada não convectiva (CNC), que tem concentração de sal decrescente à medida que se afasta do fundo do tanque. Assim, a turbulência resultaria numa diluição excessiva da CCI e aumentando sua espessura de maneira indesejada. $\mathrm{O}$ processo de encher o tanque completamente durou cerca de oito horas. Uma representação esquemática do TSGS piloto é apresentada na Figura 1.

\section{Metodologia experimental}

Após o preenchimento do tanque, o mesmo foi deixado quinze dias de repouso, sem nenhuma medida realizada, para que as camadas formadas se estabilizassem. Após esse tempo, foi iniciado o registro das temperaturas no tanque. Para isso, as medições de temperatura foram realizadas em triplicata em três diferentes alturas do tanque $(5 \mathrm{~cm}$, $35 \mathrm{~cm}$ e $65 \mathrm{~cm}$, a partir do seu fundo), determinadas a partir da observação visual das camadas CCS, CNC e CCI no interior do TSGS. A temperatura em cada altura, ou camada, foi a média das respectivas replicatas.

Para tanto, utilizou-se um termopar (Fullgauge, Brasil.) com precisão de $0,1^{\circ} \mathrm{C}$ e uma sonda metálica de $5 \mathrm{~mm}$ de diâmetro e $1 \mathrm{~m}$ de comprimento, na qual foram fixados os sensores do termopar nas alturas pré-determinadas, sendo estabelecido um padrão de tempo de imersão dos sensores de 3 minutos em cada um dos pontos.

A temperatura ambiente foi aferida na sombra, em local protegido do vento. As medições foram realizadas durante os dias 20 de agosto e 3 de novembro de 2008, em dias alternados e horários escolhidos entre 10:00 h e 17:00 h, totalizando 32 dias de registros.
Figura 1: Representação esquemática do TSGS piloto construído. A - dimensões do tanque e orientação do mesmo em relação aos pontos cardeais. B - formação das diferentes camadas no tanque e planos de medição da temperatura.

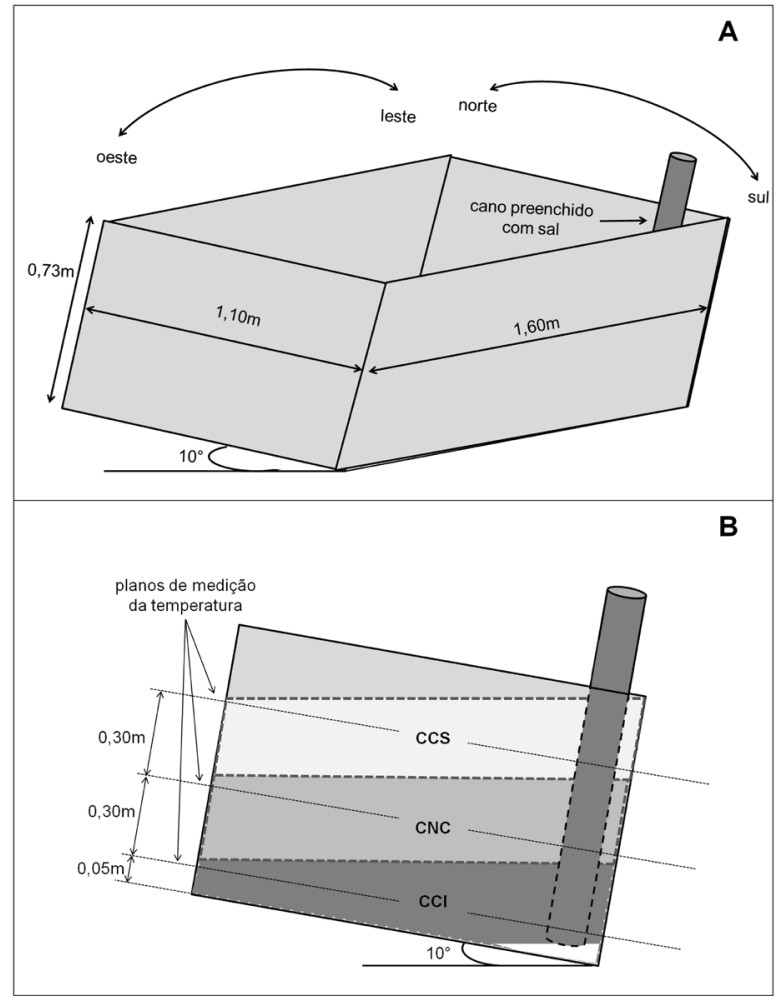

\section{Cálculo do potencial térmico do tanque solar de gradiente salino}

A partir dos valores de temperatura na CCI e das camadas superiores, foi possível estimar a quantidade média de energia armazenada na forma de calor nesta camada, por meio da Equação 1. A massa de água foi calculada por meio de uma relação matemática entre a densidade da água e o volume de água na CCI.

$$
Q=m \times c_{P} \times \Delta T
$$

Em que $Q$ é a quantidade de energia armazenada na forma de calor $(\mathrm{kJ}), \mathrm{m}$ é a massa de água $(\mathrm{kg})$ , $c_{P}$ é o calor específico $(4,185 \mathrm{~kJ} / \mathrm{kg} . \mathrm{K})$ e $\Delta T$ é a diferença de temperatura $\left(\mathrm{K}\right.$ ou $\left.{ }^{\circ} \mathrm{C}\right)$ entre a $\mathrm{CCI}$ e a CCS. 


\section{Resultados e discussão}

Na Figura 2, são apresentados os resultados médios obtidos para as temperaturas nas camadas CCI, CNC e CCS do TSGS, além da temperatura ambiente, nos 32 dias de registros entre 20 de agosto e 03 de novembro de 2008.

Figura 2: Temperatura registrada nas camadas do TSGS e no ambiente durante o período avaliado.

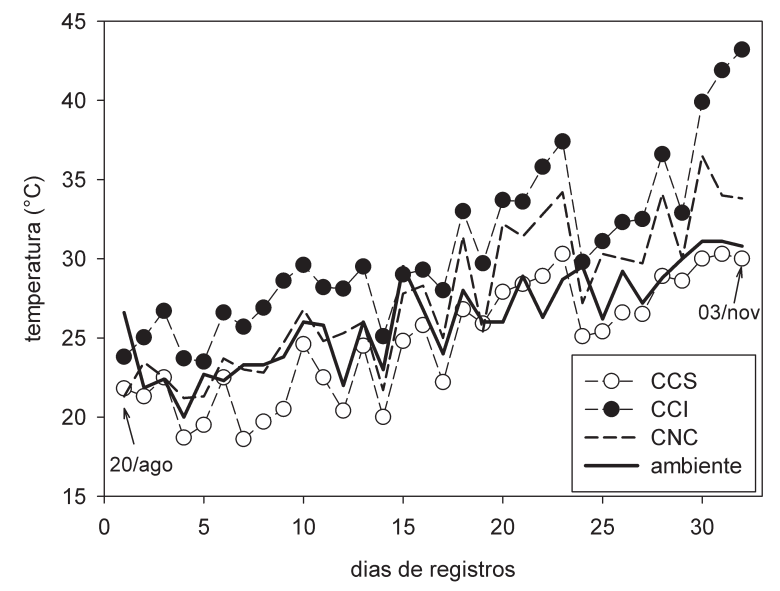

Verificou-se uma tendência no aumento da temperatura no tanque com o decorrer do tempo e a aproximação do verão. Observa-se também que nos primeiros dias em que as temperaturas foram registradas a diferença entre a CCI e a CCS foi menor que nos últimos dias, demonstrando que na CCI estava havendo um acúmulo de energia na forma de calor. Tal comportamento também indica que apenas uma pequena parte da energia armazenada na forma de calor durante o período diurno foi perdida no período noturno. Ould Dah et al. (2010) verificaram que para um TSGS com 0,9 m de diâmetro, 20 dias foram suficientes para a estabilização da temperatura na CCI.

A menor diferença de temperatura observada entre as camadas CCI e CCS foi de $2{ }^{\circ} \mathrm{C}$ no primeiro dia de registros, enquanto que a maior diferença observada foi de $13,2{ }^{\circ} \mathrm{C}$ no último dia. Em média, para o período observado, a diferença de temperatura foi de $5,70{ }^{\circ} \mathrm{C}$. Convém ressaltar que o período analisado envolve parte do inverno e início da primavera, quando a temperatura média é mais baixa. Se o experimento fosse conduzido em um período mais quente, provavelmente a diferença de temperatura média seria ainda maior, o que reforça o potencial de uso dos TSGS.

Com a diferença média obtida, o potencial energético médio armazenado na CCI foi de $7135 \mathrm{~kJ}$. Considerando-se uma eficiência em torno de 30\% na utilização da energia armazenada (LEBLANC et al., 2011), seria possível, por exemplo, aquecer em $10^{\circ} \mathrm{C}, 50 \mathrm{~L}$ de leite resfriado em um laticínios. No dia em que a maior diferença de temperatura foi registrada, a mesma quantidade de leite poderia ter sua temperatura elevada em cerca de $23^{\circ} \mathrm{C}$. Com isso, poderia haver economia na utilização de outras fontes energéticas, como por exemplo o combustível de uma caldeira.

Kumar e Kishore (1999) estudaram a construção e operação de um tanque solar em Kutch - Índia e verificaram sua aplicabilidade em processos industriais. Casos de sucesso na utilização da energia térmica armazenada em TSGS's também são relatados na Austrália e Estados Unidos (LEBLANC et al., 2011). Contudo, estudos aprofundados são necessários para avaliar a eficiência desta tecnologia no contexto nacional.

Os resultados obtidos no rudimentar tanque avaliado neste experimento corroboram com trabalhos realizados por outros autores, demonstrando o potencial que os TSGS's apresentam como fontes alternativas de energia renovável. Estudos mais aprofundados acerca da eficiência na utilização da energia armazenada e dos custos envolvidos são necessários para que tal tecnologia possa ser difundida em nosso país.

\section{Conclusões}

Os resultados obtidos neste trabalho demonstram que é possível armazenar em um TSGS energia térmica obtida a partir da energia solar. Em 
etapas posteriores essa energia térmica poderá ser convertida em outras formas de energia, de forma sustentável e não poluente.

A utilização desta tecnologia alternativa por parte das indústrias, propriedades rurais ou outras que requeiram processos de pré-aquecimento de fluidos, pode resultar em redução de custos, menor geração de poluentes e maior sustentabilidade ecológica.

\section{Referências}

AGHA, K. R.; ABUGHRES, S. M.; RAMADAN, A. M. Design methodology for a salt gradient solar pond coupled with an evaporation pond. Solar Energy, v. 72, n. 05, p. 447-454, 2002.

ALAGAO, F. B. Simulation of the transient behavior of a closed-cycle salt gradient solar pond. Solar Energy, v. 56, n. 03, p. 245-260, 1996.

ARREBOLA, F. V.; GALVÃO, M. E. F.; RONDON, V. S.; ABBUD, J. R. Processo de dessalinização utilizando piscina solar de gradiente salino. Revista Ceciliana, v. 02, n. 01, p. 49-51, 2010.

DHERE, N.; CRUZ, L. R.; LOBO, P. C.; BRANCO, J. R. T.; RUTHER, R.; ZANESCO, I.; LIMA, J. H. G. History of solar energy research in Brazil. In: Proceedings of the ISES 2005 Solar World Congress, Freiburg, Alemanha, 2005. p. 614-619.

DOR, I.; EHRUCH, A. The Effect of Salinity and Temperature Gradients on the Distribution of Littoral Microalgae in Experimental Solar Ponds, Dead Sea Area, Israel. Marine Ecology, v. 08, n. 2, p. 193-205, 1987.

GIESTAS, M. C.; JOYCE, A.; PINA, H. The influence of non-constant diffusivities on solar ponds stability. International Journal of Heat and Mass Transfer, v. 40, n. 18, p. 4379-4391, 1997.

GIESTAS, M. C.; PINA, H. L.; MILHAZES, J. P.; TAVARES, C. Solar pond modeling with density and viscosity dependent on temperature and salinity. International Journal of Heat and Mass Transfer, v. 52, n. 11-12, p. 2849-2857, 2009.

HASNAIN, S. M. Review on sustainable thermal energy storage technologies, Part I: heat storage materials and techniques. Energy Conversion and Management, v. 39, n. 11, p. 1127-1138, 1998.

HULL, J. R. Membrane stratified solar ponds. Solar Energy, v. 25, n. 04, p. 317-325, 1980.

INMET. Instituto Nacional de Meteorologia. Dados meteorológicos da estação automática de Itapetinga BA. Disponível em $<$ www.inmet.gov.br $>$. Acessado em: 20/06/2012.

JANNUZZI, G. M. Políticas públicas para eficiência energética e energia renovável no novo contexto de mercado: uma análise de experiência recente dos EUA e do Brasil. 1. ed. Campinas: Autores Associados, 2000. p. 45-57.

JORGE, J. R. A.; FADIGAS, E. A. F. A. Estudo da viabilidade de instalação de piscinas solares para produção de energia elétrica. Seleção Documental: Inteligência Artificial e Novas Tecnologias, v. 22, p. 5-11. 2011.

KISHORE, V. V. N.; KUMAR, A. Solar pond: an exercise in development of indigenous technology at Kutch, India. Energy for Sustainable Development, v. 3, n. 1, p. 17-28, 1996.

KUMAR, A.; KISHORE, V. V. N. Construction and operational experience of a $6000 \mathrm{~m} 2$ solar pond at Kutch, India. Solar Energy, v. 65, n. 04, p. 237-249, 1999.

LEBLANC, J.; AKBARZADEH, A.; ANDREWS, J.; LU, H.; GOLDING, P. Heat extraction methods from salinity-gradient solar ponds and introduction of a novel system of heat extraction for improved efficiency. Solar Energy, v. 85, n. 12, p. 3103-3142, 2011.

LOBO, P. C.; ARAÚJO Jr., L. U. Performance of a model shallow salt gradient solar pond. In: Proceedings of ENERGEX'84, Regina, Canadá. 1984. P. 551-555.

LU, H.; SWIFT, A. H. P.; HEIN JR., H. D.; WALTON, J. C. Advancements in Salinity Gradient Solar Pond Technology Bases on Sixteen Years of Operational Experience. Journal of Solar Energy Engineering, v. 126, n. 06, p. 759-768, 2004.

MALIK, N.; DATE, A.; LEBLANC, J.; AKBARZADEH, A.; MEEHAN, B. Monitoring and maintaining the water clarity of salinity gradient solar ponds. Solar Energy, v. 85, n. 11, p. 2987-2996, 2011.

OULD DAH, M. M.; OUNI, M.; GUIZANI, A.; BELGHITH, A. The influence of the heat extraction mode on the performance and stability of a mini solar pond. Applied Energy, v. 87, n. 10, p. 3005-3010, 2010.

OUNI, M.; GUIZANI, A.; LU, H.; BELGHITH, A. Simulation of the control of a salt gradient solar pond in the south of Tunisia. Solar Energy, v. 75, n. 1, p. 95-101, 2003.

RABL, A. Active Solar Collectors and Their Applications. 1st ed. New York: Oxford University Press, Inc., 1985, p. 5-13. 
RIFFE, D. B.; LEITE, A. P. F.; BELO, F. A. Simulação do aporte térmico de coletores solares planos em um tanque cilíndrico estratificado. In: I Congresso Brasileiro de Energia Solar, Fortaleza, Brasil: Associação Brasileira de Energia Solar (ABENS), 2007. Disponível em: <http:// dougbr.webng.com/artigos/riffel07a.pdf $>$. Acesso em: 25 mar. 2011.

SHAH, S. A.; SHORT, T. H.; FYNN, R. P. Modeling and testing a salt gradient solar pond in northeast Ohio. Solar Energy, v. 27, n. 05, p. 393-401, 1981.

TABOR, H. Solar ponds. Solar Energy, v. 27, n. 03, p. 181-194, 1981.

TUNDEE, S.; TERDTOON, $\quad$ P.; SAKULCHANGSATJATAI, P.; SINGH, R.; AKBARZADEH, A. Heat extraction from salinity. Solar Energy, v. 84, n. 9, p. 1706-1716, 2010.

VELMURUGAN, V.; SRITHAR, K. Prospects and scopes of solar pond: A detailed review. Renewable and Sustainable Energy Reviews, v. 12, n. 8, p. 2253-2263, 2008.

Recebido em 28 Setembro 2011-Received on September 28, 2011. Aceito em 25 Julho, 2012 - Accepted on July 25, 2012. 
Veras, D. V. S. et al. 